\title{
Dynamic communication for smart metering : leading edge for sustainable energy and PHEV
}

\begin{abstract}
The urgency of moving towards optimization of existing energy distribution via Smart Grid (SG) is crucial due to promising opportunities such as energy efficiency, reduced carbon emissions, and improved power reliability. This paper focuses on wireless communication network for smart meter linking Information and Communication Technologies (ICT) to Smart Grid (SG). This paper also gives evidence that before smart meter is deployed, its communication infrastructure has to be precise to ensure proper operation, functionality and safety. This paper also provides an overview of how smart meter can contribute the penetration of Plug-In Hybrid Electrical Vehicle (PHEV) to the grid and its uncertain load.
\end{abstract}

Keyword: Smart grid; Communication network; Smart meter; PHEV 